\title{
L'infâme derrière des ci-devantsLe cul aristocrate et la contre-révolution
}

The Aristocratic " arse " and the counterrevolution

\section{Stéphanie Genand}

\section{(2) OpenEdition \\ Journals}

Édition électronique

URL : https://journals.openedition.org/ahrf/11716

DOI : 10.4000/ahrf.11716

ISSN : 1952-403X

\section{Éditeur :}

Armand Colin, Société des études robespierristes

Édition imprimée

Date de publication : 1 septembre 2010

Pagination : 145-156

ISBN : 978-2-200-92633-5

ISSN : 0003-4436

Référence électronique

Stéphanie Genand, "L'infâme derrière des ci-devantsLe cul aristocrate et la contre-révolution », Annales historiques de la Révolution française [En ligne], 361 | juillet-septembre 2010, mis en ligne le 01 septembre 2013, consulté le 23 avril 2022. URL : http://journals.openedition.org/ahrf/11716 ; DOI : https://doi.org/10.4000/ahrf.11716 


\title{
L'INFÂME DERRIÈRE DES CI-DEVANTS LE CUL ARISTOCRATE ET LA CONTRE-RÉVOLUTION
}

\author{
Stéphanie GENAND
}

\begin{abstract}
La Révolution associe la régénération nationale à l'exclusion de l'aristocratie. Ce groupe, considéré comme l'ennemi du régime, suscite une haine qui dépasse la légitimité des motifs idéologiques. Le noble incarne une altérité monstrueuse, inscrite dans un processus de déshumanisation et d'infamie, qui s'appuie sur le double registre de représentation du «cul » sous l'Ancien Régime. À la fois espace intime protégé des regards et surface qui dissimule une profondeur inquiétante, le « cul » occupe le centre des réquisitoires qui appellent à chasser ou massacrer les aristocrates. Cette valeur symbolique nourrit un discours d'exclusion plus radical que les condamnations de pratiques sexuelles contre-nature: le cul devient le lieu d'une sanction identitaire et non plus circonstancielle ou politique.
\end{abstract}

Mots-clés : cul, aristocratie, infâme, flétrissure, profondeurs.

Parmi les armes qui nourrissent la Révolution et fondent sa légitimité, l'exclusion constitue sans doute la plus efficace et la plus stratégique. La désignation de l'ennemi, dressant une frontière entre patriotes et réactionnaires, unifie par le morcellement l'élan de la régénération nationale. À l'énergie constructive, qui invite le pays à la métamorphose, répond souterrainement l'écho des haines assassines : diviser, dénoncer et chasser réécrivent en lettres menaçantes la liberté, l'égalité et la fraternité. Un tel clivage, loin de constituer une spécificité de la Révolution française, structure chaque épisode de la redéfinition des identités dans l'histoire : l'angoisse de l'avenir s'appuie sur la théâtralisation du congé signifié au passé. La chute de l'Ancien Régime, qui marque une rupture sans précédent, aggrave en la radicalisant cette tendance à la séparation. La nation se construit dans le refus d'un dangereux ennemi. 
L'aristocratie, à qui revient explicitement cette fonction dans les pamphlets, cumule les griefs qui légitiment le rejet politique dont elle fait l'objet. Privilèges, monopoles, soutien au roi et préparation d'un complot, les chefs d'accusation abondent, qui alimentent la haine jacobine et les mesures de répression votées sous la Terreur. Ce réquisitoire, s'il relève de l'arsenal révolutionnaire, surprend pourtant par une virulence extraordinaire et troublante. Le noble, à lire les nombreuses invectives qu'il attire, ne représente pas seulement le passé monarchiste et la réaction idéologique. Il incarne une altérité répugnante, à la frontière de l'humanité, associée à cette partie de l'anatomie aussi symbolique qu'inquiétante : le cul.

Cette focalisation s'inscrit dans la représentation problématique du corps sous l'Ancien Régime. L'article signé par d'Alembert dans l'Encyclopédie souligne le principe de fragmentation qui prévaut malgré les progrès de la médecine : "L'anatomie étant une espèce de géographie dans laquelle la précision est nécessaire, on a divisé le corps, comme la terre, en plusieurs régions ${ }^{1}$. Le morcellement, s'il traduit la difficile appréhension de la matière, invite à la concevoir comme une juxtaposition de territoires indépendants. Le cul se voit dès lors associé à l'arrière et à la profondeur. La définition du Dictionnaire de l'Académie reprend, en 1798, celle donnée par l'Encyclopédie: "Le derrière, cette partie de l'homme qui comprend les fesses et le fondement ». La surface anatomique recouvre ainsi un espace à la fois dissimulé et opaque. Cette dualité, loin de désarmer les contemporains, nourrit au contraire deux registres de représentations : le visible, offert sous la forme d'une chair tendre et d'une peau claire, et l'invisible, lié aux mystères de l'anal et de l'excrémentiel. La haine de la noblesse, qui outrepasse la légitimité des motifs politiques, croise cette ambivalence et voue le cul à une double exclusion : intime à profaner ou théâtre de l'abject, il incarne, sous la Révolution, l'horreur fascinée du corps infâme.

Ce mot appartient au registre des condamnations juridiques de l'Ancien Régime. "Infâme » désigne, dans le Dictionnaire de l'Académie, la personne « flétrie par les lois et par l'opinion publique $»^{2}$. La flétrissure, elle-même associée au châtiment corporel, renvoie à l'acte de « condamn[er] [un homme] à être marqué d'un fer chaud » ${ }^{3}$. Au dis-

(1) Denis Diderot, Encyclopédie ou Dictionnaire raisonné des sciences, des arts et des métiers, Paris-Neuchâtel, Briasson-David, 1751-1765, t. IV, p. 264.

(2) Dictionnaire de l'Académie, édition de 1798.

(3) Ibidem. 
crédit de la réputation s'ajoute, dès le XIV ${ }^{\mathrm{e}}$ siècle, l'imposition d'une marque considérée comme un signe honteux et indélébile. Cette trace, qui condamne le criminel à ne pouvoir cacher l'identité flétrie, transforme la peau en parchemin témoin des turpitudes. Sa tendresse et sa valeur symbolique, à mi chemin entre la surface et l'être intime, offrent un espace privilégié à la trace infamante de la justice. La question se pose alors du choix de la partie du corps propice à l'empreinte sacrilège. Le cul attire par une blancheur marquée qui le distingue et attire le regard. Lorsqu'il retrace les pérégrinations de la belle Léonore à travers l'Afrique, Sade souligne, non sans effet comique, la fascination exercée par le « local» de son héroïne menacée de mourir empalée. Contrainte de se teindre la peau en noir pour échapper à ses poursuivants, elle provoque avec son corps bicolore la frayeur et la consternation des spectateurs :

« Pour l'accomplissement de cette cérémonie, à peu près comme pour celle où l'on châtie les enfants, la portion de chair que l'on découvre est celle que la nature a placée au bas de nos reins; et cela, pour que rien ne puisse mettre obstacle à l'introduction du pieu dans la partie destinée au supplice. On dégarnit donc promptement aux yeux du monarque observateur ce qui gênait dans moi le local nécessaire à l'action. [...] Trop émue de mon sort, je n'avais pas pensé à la surprise que je devais naturellement causer en présentant un derrière assez blanc sous un buste fort noir; la frayeur avait été générale; les uns m'avaient prise pour un dieu, les autres pour un sorcier, mais tous s'étaient enfuis $»^{4}$.

Cet épisode, malgré sa dimension parodique, souligne la singularité du « bas des reins » dans la scène du supplice. Le cul, traditionnellement dissimulé aux regards, apparaît dans un geste de désacralisation des corps invisibles. Femmes et nobles, comme le rappelle Mercier dans le chapitre consacré aux « latrines » du Tableau de Paris, partagent le secret de la pudeur et du mystère intime :

«Les femmes sur ce point sont plus patientes que les hommes; elles savent si bien prendre leurs mesures, que la plus dévergondée ne donne jamais le spectacle qu'offre en pleine rue l'homme réputé chaste. Les observations désirées des médecins, si un jour elles avaient lieu, ne pour-

(4) Donatien-Alphonse François marquis de $\mathrm{SADE}$, Aline et Valcour ou le roman philosophique, 1795, réed. M. Delon, Paris, Gallimard, t. I, 1990, p. 769. 
raient déterminer, d'après la notoriété publique dont nous parlons, que les tempéraments masculins; il faudrait recourir ailleurs pour constater celui des femmes $»^{5}$.

La mise à nu du cul, lorsqu'elle ouvre le cérémonial du châtiment, impose une révélation qui abolit la frontière du privé.

Cette première sanction, en forme de profanation, se prolonge dans l'imposition d'une flétrissure qui marque le criminel. En marge de la guillotine et de ses vertus égalitaristes, la Révolution réserve aux aristocrates le privilège de la trace infamante. Si le fer a disparu des pratiques judiciaires de l'époque, la fessée, sur un mode mineur, se charge à son tour de valeurs symboliques. Son caractère humiliant efface le prestige social et infantilise la victime en lui rappelant l'inversion qui prévaut : à la hiérarchie pyramidale de l'Ancien Régime, elle substitue le royaume à l'envers du bas en haut et du cul par-dessus tête. Parmi les cibles désignées de ce carnaval, la noblesse dispute au clergé le sacrilège des fesses molestées. L'anecdote, qui frappe en 1790 la célèbre figure de l'abbé Maury ${ }^{6}$, inspire de nombreux récits sur le modèle de Faites beau cul, vous n'aurez

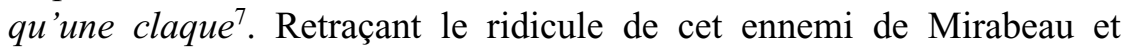
du tiers état, ce pamphlet déroule les étapes d'une déchéance d'autant plus humiliante qu'elle intervient dans la cour d'un collège. Après avoir échappé de justesse à la lanterne, l'abbé Maury se voit contraint de se dévêtir pour un châtiment corporel :

«Mais ayant pris la voix de la douceur, et sur les représentations que l'abbé Maury était personne sacrée, comme membre de l'assemblée nationale. "Alors les écoliers ont tenu conseil, et sur l'observation que fit leur président, que l'assemblée n'avait pas défendu de donner le fouet à ses membres aristocrates, on est allé aux voix et le jugement a été rendu en ces termes." L'abbé Maury déclaré atteint et convaincu d'être traître à la patrie, pour réparation de quoi, est condamné à faire amende honorable aux portes principales de la cour du Collège, culotte bas, où il recevra, de la main de Painblanc, trois coups de fouet. Lequel jugement a été exécuté selon sa forme et sa teneur. On observe qu'au moment de l'exécution,

(5) Louis Sébastien Mercier, Tableau de Paris, 1781-1789, réed. J.-C. Bonnet, Paris, Mercure de France, t. I, 1994, p. 176.

(6) Voir sur ce sujet Ouzi Elyada, «La mise au pilori de l'abbé Maury: imaginaire comique et mythe de l'antihéros pendant la Révolution française », $A H R F, \mathrm{n}^{\circ}$ 341, 2005, p. 1-24.

(7) Faites beau cul, vous n'aurez qu'une claque, ou Événement arrivé à l'abbé Maury, dans la cour du Collège Mazarin, par les écoliers, le 27 à trois heures, Paris, 1790. 
ledit abbé faisant des difficultés, Painblanc lui a dit : “Allons, M. l'Abbé, faites beau cul, vous n'aurez qu'une claque." Il s'y est soumis $»^{8}$.

Aux vertus libératoires de la fessée d'une «personne sacrée » s'ajoute l' « amende honorable » qui relègue le prêtre au rang des infâmes. Le bourreau, symboliquement affublé du surnom de «Painblanc», consomme dans son geste la subversion du programme révolutionnaire.

Frapper le cul devient alors le fer de lance de la propagande antinobles. S'il n'est pas toujours évoqué de manière aussi détaillée, le geste inspire plusieurs titres désireux d'exploiter son potentiel de violence. La lutte contre l'ennemi exige le ralliement des patriotes pour chasser physiquement les scélérats. Le Passeport des capucins ou le Chapitre des coups de pied dans le cul, pour servir d'éclaircissement aux aristocrates ${ }^{9}$, témoigne de l'importance politique de cette partie du corps. La force de l'image, qui transforme la fessée en « coup de pied », suggère une gradation qui conduit aux diatribes ordurières du Père Duchêne :

« Mille millions d'aristocrates! Que je suis donc content! Camarade Lafleur, ne m'a-t-il pas dit ce matin en tordant notre sacrée chopine, que tous les parlements sont foutus? Ah la sacrée canaille! Je me fous aujourd'hui en ribotte : ces chiens de gueux ont tant bougraillé la France, qu'à la fin on leur a foutu de la pelle au cul $»^{10} \ldots$

L'expression « donner la pelle au cul $»{ }^{11}$, récurrente dans les textes de l'époque, ajoute au châtiment corporel la nécessaire expulsion de la noblesse. La marque humiliante, première étape de la mise au ban, se prolonge dans une traque qui interdit le territoire aux « ci-devants ». Si la fessée s'attaquait à une surface protégée par un luxe obscène, la «pelle » déshumanise en transformant l'aristocrate en déchet. Animal nocturne, insecte nuisible ou bête ne se déplaçant qu'en troupeaux, il évoque des hordes qu'il faut éradiquer au nom de la patrie :

«Plus de vingt autres conjurés, introduits par le bas valet Thierry, viennent à la faveur de la nuit, se rendre à l'Assemblée diabolique. Leurs yeux étincelants de rage, sont les seules lumières qui éclairent ces espèces

(8) Ibidem, p. 2.

(9) Le Passeport des capucins, ou le chapitre des coups de pied dans le cul, pour servir d'éclaircissement aux aristocrates, Paris, 1790.

(10) Le Trou du cul du Père Duchesne, ou le mouchoir des aristocrates, Paris, 1790, p. 3.

(11) Voir dans ce numéro l'article de Michel Biard et Jacques Guilhaumou sur l'importance de cette expression linguistique. 
des sabbats nocturnes. Ces proscrits se partagent par pelotons pour délibérer sur les moyens de perdre leur patrie et leur Roi. [...] D'autre part, disent certains cabalistes, ces démoniaques accumulant les crimes, invoquent les puissances infernales, leur dressent des autels, et leur font des prières, des sacrifices et des offrandes sacrilèges, pour obtenir la destruction de cet Empire $»^{12}$.

Cette hantise du complot, traditionnellement qualifié de «sabbat $»^{13}$, alimente un réquisitoire qui exclut l'ennemi du genre humain. L'Accusateur public de Richer-Serisy, à la délicate question de l'identification physique du noble, répond par un autoportrait où l'outrance le dispute au monstrueux :

« Je suis donc un aristocrate, dites-vous, parlez! À quels traits pouvezvous me reconnaître? [...] Les aristocrates sont ceux qui [...] osent de ce cloaque impur insulter effrontément l'homme de bien qui gémit isolé sur cette terre sanglante et dépouillée, et se contente d'offrir quelquefois à ses concitoyens, qui les accueillent, ces feuilles fugitives et baignées de ses larmes. Les aristocrates sont ces vers engendrés du cadavre des factions qui veulent se réunir en une masse putride pour peser sur la Convention régénérée, l'entraîner à sa perte, et nous enlever, par leur stupidité et leur orgueil plus sot encore, les faibles ressources qui restent à la patrie expirante $\rangle^{14}$.

La métaphore de l'organisme corrompu, si elle structure le discours révolutionnaire ${ }^{15}$, s'aggrave ici d'une spatialisation qui laisse entendre une parole venue du « cloaque ». Cette peur de l'égout, dont remonterait l'immonde des profondeurs, radicalise la différence qui sépare 1'aristocrate de la nation. Il ne s'agit plus seulement de retourner les prérogatives sociales et financières : le privilégié devient cette altérité condamnée aux marges et aux souterrains.

Une telle exclusion, reliant la noblesse à l'obscurité du «derrière », impose à son action politique la clandestinité et la logique de

(12) La Ligue aristocratique, ou les Catilinaires françaises, par un membre du Comité patriotique du caveau, au Palais royal, Paris, 1789, p. 4.

(13) Voir notamment Le Passeport des capucins, p. 1 : «Grâces au redoutable gardien des capucins, qui a donné du pied au cul au sabbat qui se tenait dans son couvent, la noire cohorte s'est enfin dissipée...».

(14) Charles-Pierre Ducancel, Richer-SERIsy, L'Accusateur public, Paris, 1797, vol. I, p. 4.

(15) Voir Stéphanie GENAND, «Eros politique. Idéologies du corps à la fin de l'Ancien Régime », Dix-huitième siècle, n 37, 2005, p. 577-597. 
l'inversion. La contre-révolution, conformément aux valeurs du préfixe, avance à rebours de toute dynamique nationale. Aux rassemblements des armées dans le secret des zones frontalières, elle ajoute le projet d'imposer le retour aux forces de jadis. Cette réaction, qui menace l'élan de la régénération, inquiète d'autant plus qu'elle opère de manière invisible ou masquée. L'aristocrate, malgré la haine virulente qu'il suscite dans les discours, parvient le plus souvent à dissimuler sa véritable identité. La menace d'une réapparition de l'Ancien Régime aboli réactive alors le cauchemar d'une remontée en surface du « cloaque ». Les nobles, répandus dans les rues de Paris, se mêleraient à la population pour accélérer la contagion régressive. À l'image du virus et de la maladie des « corps vénéneux $»^{16}$ se superpose celle de l'excrément inscrit dans la couleur choisie comme marque infamante :

« Que fera-t-on, lorsque la contre-révolution sera arrivée, à un Lecoin, dont le caractère horrible et les assertions calomnieuses sont affichées aujourd'hui sur tous les murs de Paris, et dont la scélératesse a ensanglanté le palais de Versailles. Âmes pures, gardez-vous de souiller vos mains dans le sein de ce monstre; substituons à l'affront du bonnet vert, une marque flétrissante, digne de ce monstre régicide; qu'il soit traîné dans tout Paris, affublé d'une énorme houppelande boue de Paris et caca dauphin $»^{17}$.

La contre-révolution doit arborer un signe à la hauteur de ses ambitions. Caca dauphin devient la cocarde infernale de ce monde des profondeurs, qui revendique le droit d'imposer le règne du frivole et du libertinage :

« Mais le comble du despotisme amène la délivrance. Tremblez, nos maîtres! Il n'est point de milieu; ou épurez vos goûts, ou nous brisons nos chaînes. L'époque du fichu et du pet-en-l'air avait déjà ébranlé votre empire : le temps et les cheveux à la reine achevaient de ramener les esprits; mais le déluge de merdes d'oies, de caca dauphin et de boue de Paris, dont vous inondâtes l'Europe, n'est pas encore écoulé : il croupit et fermente sourdement. On murmure du fond du cloaque; on crie à la tyrannie; on se rappelle sa dignité. Le désespoir touche à l'héroïsme :

(16) La Ligue aristocratique, p. 6 : «Vous mériteriez qu'on arrachât vos cœurs corrompus, et qu'on traînât dans la fange, les corps vénéneux que vous avez déjà plongés dans les vices ».

(17) Guillaume-Marie-Anne Brune, Journal de la Cour et de la ville, Paris, 1792, t. I, p. 379 . 
un seul éclair peut embraser le feu de la révolte, et nous touchons peutêtre à quelque grande révolution. Indépendance sera la devise de nos bannières, caca dauphin notre cri de guerre, et simplicité notre mot de ralliement $»^{18}$.

Les Principes philosophiques de Weiss résonnent ici du murmure d'un monde souterrain : le «fond du cloaque » fomente une subversion écrite en lettres de boue et de déchets immondes.

Cette métaphore anale appartient au langage de la Révolution. Le chapitre du Tableau de Paris consacré aux « latrines » souligne l'instabilité d'une capitale menacée de submersion par la surcharge des évacuations :

«Les trois quarts des latrines sont sales, horribles, dégoûtantes : les Parisiens, à cet égard, ont l'œil et l'odorat accoutumés aux saletés. Les architectes, gênés par l'étroit emplacement des maisons, ont jeté leurs tuyaux au hasard, et rien ne doit plus étonner l'étranger, que de voir un amphithéâtre de latrines perchées les unes sur les autres, contiguës aux escaliers, à côté des portes, tout près des cuisines, et exhalant de toutes parts l'odeur la plus fétide. Les tuyaux trop étroits s'engorgent facilement; on ne les débouche pas; les matières fécales s'amoncellent en colonnes, s'approchent du siège d'aisance; le tuyau surchargé crève; la maison est inondée; l'infection se répand, mais personne ne déserte : les nez parisiens sont aguerris à ces revers empoisonnés. Que ceux qui ont soin de leur santé, ne jettent jamais leurs excréments chauds dans ces trous qu'on appelle latrines, et qu'ils n'aillent point offrir leur anus entrouvert à ces courants d'air pestilentiels; mieux vaudrait y mettre la bouche, car l'acide de l'estomac les corrigerait $\gg{ }^{19}$.

La puissance de ce torrent excrémentiel, qui gronde dans le sol de Paris, expose chaque quartier au risque d'un jaillissement des profondeurs. Mercier, avant de devenir un membre actif de la Convention nationale, décrit le danger des «latrines » comme le spectacle d'une inversion des hiérarchies. Le souterrain, dont l'énergie finit par percer la surface, prend des allures de foule malodorante reléguée aux marges des « faubourgs ». Le quartier Saint-Marcel, rempli d'une « rumeur épou-

(18) Franz-Rudolph von WeIss, Principes philosophiques, politiques et moraux, en Suisse et à Paris, Maradan, 1789, t. II, p. 88.

(19) Louis-Sébastien Mercier, Tableau de Paris, t. II, p. 1071. 
vantable », dégage une « odeur infecte $»^{20}$ qui éloigne de la « populace ». Ces images, loin d'inviter au mépris pour le bas peuple, nourrissent chez Mercier la fibre humaniste et l'appel au progrès. Les administrateurs de la capitale, exaspérés par l'échafaudage des latrines, doivent substituer à ces cloisons illusoires les vertus du plein air et des quais. Le Tableau de Paris, fidèle aux préceptes « aéristes », souligne les bienfaits des espaces publics de soulagement :

« Les personnes les plus propres et les plus délicates, dont l'imagination est toujours fleurie, ne vivant point avec ces hommes impolis, qui satisfont grossièrement les besoins de la nature, les repoussant même loin d'elles et de leur société, sont obligées néanmoins de communiquer par la vue avec ce qu'ils déposent en plein air. Les excréments du peuple avec leurs diverses configurations sont incessamment sous les yeux des duchesses, des marquises et des princesses. Ô quelle moralité n'y aurait-il pas à faire là-dessus! $»^{21}$

Ces latrines naturelles révèlent une égalité qui échappe aux mensonges et aux scandales injustes des logements. Étalés au grand jour, les immondices se banalisent et rappellent l'arbitraire des notions d'ignoble et de vulgaire.

La Révolution vient pourtant bouleverser cette concorde éphémère. L'excrément, s'il relativise le poids des hiérarchies, se transforme en symptôme de noirceur aristocratique. Cette inversion s'explique peut-être par le retour d'une ségrégation identitaire. Si la Terreur engendre un arsenal de mesures destinées à radier et tuer, selon le mécanisme de revanche analysé par Bronislaw Baczko ${ }^{22}$, cette distinction rétablit l'existence d'un corps rétif au programme de l'universel. Cette différence, loin de marquer la limite du projet révolutionnaire, réactive au contraire le fondement de l'aristocratie :

«Qu'est-ce d'abord que la noblesse sinon une altérité? De pays à pays, la définition même de la noblesse, quand elle est univoque, ce qui est rare, varie. La pratique nobiliaire est, de l'est de l'Europe à l'ouest du continent, si diverse qu'elle représente, à la vérité, des degrés d'évolution historique distants de plusieurs siècles. Parmi ces divergences, une seule

(20) Ibidem, t. I, p. 219.

(21) Ibidem, t. II, p. 176.

(22) Bronislaw BaczKo, Politiques de la Révolution française, Paris, Gallimard, 2008. 
convergence : la notion de noblesse inclut un certain nombre de gens, mais exclut la grande majorité du pays $»^{23}$.

Liée par un sang unique dont l'origine se confond avec celle du pays, la noblesse a traditionnellement établi son identité sur le principe de l'altérité. La Révolution, qui renverse les codes de l'Ancien Régime, transforme cette élection en motif de malédiction : la supériorité légitime une politique d'exclusion sélective, à rebours des articles préliminaires de la Déclaration de 1789. La violence de cette infamie explique l'acharnement contre un ennemi coupable de crimes inaccessibles aux autres citoyens. L'image de l'excrément, du déchet comme le «coup de pied au cul », singularise un réquisitoire affaibli par les contradictions qui entourent les pratiques sexuelles. Le discours contre la sodomie inspire de nombreux pamphlets qui dénoncent la prédilection aristocratique pour ce plaisir contraire à la nature. Le marquis de Villette, La Fayette ou le comte d'Artois composent la galerie des amateurs nommés dans une tradition qui associe obscène et délation :

«Dans les siècles modernes, les plus grands potentats étaient des pédérastes. Frédéric II, roi de Prusse; si célèbre par sa valeur et son génie, n'aimait pas les femmes, il enculait les hommes, et s'en faisait enculer; témoin Baculard d'Arnaud qu'il appelait son berger, et avec qui il gagna la cristalline, qu'il lui rendit bien. Témoin le roi de Suède régnant, qui fit venir dans ses états le comédien Monvel, enculeur si famé, mais qu'il renvoya après l'avoir usé et empoisonné par des assauts postérieurs. J'ai enculé moi-même la plus grande partie de mes subalternes dans les communes de la municipalité. J'ai foutu et refoutu en cul cent fois ce gredin de Mitouflet, mon procureur syndic, Vauvilliers, Blondel, Desfaucherets, Duport du Tertre $»^{24}$.

L'argument du naturel, s'il structure l'imaginaire érotique de la Révolution $^{25}$, oppose l'ardeur du patriote à la dépravation du corps flétri. Ce crime sodomite, associé à la réaction nobiliaire et au complot, se heurte

(23) Jean Meyer, « Noblesse et racisme », dans Léon Poliakov (dir.), Ni juif ni grec, entretiens sur le racisme, Actes du colloque tenu du 16 au 20 juin 1975 au Centre culturel international de Cerisy-la-Salle, Paris, Mouton, 1978, p. 113.

(24) Bordel patriotique, institué par la Reine des Français, pour les plaisirs des députés à la nouvelle législature, précédé d'une épître dédicatoire de sa Majesté à ces nouveaux Lycurgues, Paris, aux Tuileries et les marchands d'ouvrages galants, 1791, p. 40.

(25) Voir Jean-Marie Roulın, « Virilité et pouvoir dans l'imaginaire des textes érotiques de la Révolution », Itinéraires, $n^{\circ}$ 2, 2008, p. 107-121. 
pourtant à la proclamation d'une liberté des pratiques et des mœurs. Le Bordel patriotique, sous couvert de juxtaposer les scènes lascives, invite le personnel politique à découvrir l'égale valeur des plaisirs :

«Toutes les passions, tous les goûts des deux sexes y seront pleinement satisfaits; un homme y jouira avec un homme, et une femme avec une femme. Les désirs, les transports des sens y seront assouvis. Après la jouissance naturelle, la conjonction de l'homme avec la femme, on pourra librement passer à la jouissance antiphysique et comparer ensuite la différence des sensations $»^{26}$.

La figure de Théroigne de Méricourt, chargée de la direction des lieux, garantit la cohésion sociale et l'harmonie des rencontres entre les clients. Aux ébats «contre-nature » des sommités de l'aristocratie répondent, à quelques pages d'écart, les folies de Danton et Marat. Cette hétérogénéité, qui sert surtout l'objectif commercial du pamphlet, nuance le tableau d'une noblesse seule coupable de pratiques sulfureuses. Les représentations de la sexualité, traversées par les tensions politiques, n'offrent pas une scène univoque où fonder le rejet des élites. Le danger de la contre-révolution, corollaire de l'émigration, requiert un motif d'exclusion plus puissant que l'égalité qui prévaut désormais.

Le cul apparaît ainsi comme un espace symbolique et stratégique. L'ambivalence de sa définition, associant surface et profondeur, offre un double registre de représentations au discours d'exclusion qui sous-tend la Révolution. L'aristocratie, désignée comme l'ennemi monstrueux du régime, se voit condamnée à la flétrissure et au monde infâme des profondeurs.

Stéphanie GENAND

CEREDI- Université de Rouen

1, Rue Thomas Becket

76821 Mont-Saint-Aignan Cédex stephanie.genand@univ-rouen.fr 